\title{
Treating the case of incurable hysteresis in VO2
}

\author{
M. Gurvitch ${ }^{\mathrm{a}, \mathrm{b}}$, S. Luryi ${ }^{\mathrm{a}, \mathrm{c}}$, A. Polyakov ${ }^{\mathrm{a}}$, A. Shabalov ${ }^{\mathrm{a}}$ \\ ${ }^{\mathrm{a}} N Y$ State Center for Advanced Sensor Technology (Sensor CAT), ${ }^{\mathrm{b}}$ Dept. of \\ Physics and Astronomy, \\ ${ }^{\mathrm{c}}$ Dept. of Electrical and Computer Engineering SUNY at Stony Brook, Stony \\ Brook, NY 11794, U.S.A.
}

\section{Introduction}

Phase transitions present an opportunity for a useful application utilizing a natural proximity of two phases with different physical properties and an ability to trigger the transition by varying a transition-controlling parameter, such as temperature, or magnetic field, etc. There exist applications based on magnetic transitions, optical transitions, metal-insulator transitions, superconducting transitions. Some of these phase transitions, especially those classified as first order transitions (i.e. accompanied by the release or absorption of latent heat), are hysteretic in nature, so that the two phases transform into each other along different routes depending on the direction of change of a transition-controlling parameter. The hysteresis can be essential for an application. A well known example (in this case, of a hysteretic second order transition) is a ferromagnetic material which can be magnetized in an external magnetic field and, because of the hysteresis, will remain magnetized when the external field is reduced to zero. This is used for making permanent magnets and in magnetic storage of information. The stored, "memorized" magnetization can be erased, which requires specific steps to be taken, such as going around the magnetic hysteresis loop with diminishing external fields. In other cases, however, the utilization of a phase transition is hindered by its hysteresis. The "memory" of the previous history that is beneficial in magnetic materials may become detrimental in other applications, where the irreversible (or, more precisely, not easily reversible) change in a given material's property, e.g. resistivity, may be undesirable.

In this paper we consider $\mathrm{VO}_{2}$, a material undergoing first order phase transition, and refer to an application -- IR visualization utilizing resistive bolometers made of $\mathrm{VO}_{2}$-- in which hysteresis causes problems. We offer a way of operation which circumvents these problems. The applied aspects of IR visualization vis-à-vis our proposed approach are discussed in our recent publication ${ }^{[1]}$, and we will refer to it in a number of instances for further details and references. Here we want to explain the essence of the method and touch upon its physics fundamentals.

\section{Hysteretic semiconductor-metal phase transition in $\mathrm{VO}_{2}$; major and minor loops.}

In $\mathrm{VO}_{2}$ resistivity changes by 3-5 orders of magnitude in a spectacular semiconductor-to-metal phase transition around $68 \mathrm{C}^{[2]}$. In addition to resistivity, 
other properties change in this 1-st order phase transition, including crystalline structure and optical constants. In single crystals the transition is very sharp, with hysteresis width of only $1-2{ }^{\circ} \mathrm{C}^{[3]}$; however, in polycrystalline films it is broader, with hysteresis width (as measured in the middle of a transition) of $10-20{ }^{\circ} \mathrm{C}$. The resistive transition measured in one of our $\mathrm{VO}_{2}$ films can be seen in Figs. 1-4 (look at the outside major loop; ignore for now the inner or minor loops or their parts which are shown on these figures). The films used in these measurements were prepared on $\mathrm{Si} / \mathrm{SiO}_{2}$ substrates by Pulsed Laser Deposition; details of sample preparation and measurements can be found in Ref. 1. On the $\log (\mathrm{R})$ vs. T plot, starting from room temperature, with increasing $\mathrm{T}$ resistivity first follows the semiconducting S-phase slope, then falls sharply along the right (heating) branch of the transition reaching metallic M-phase; the decreasing temperature corresponds to values of $\mathrm{R}$ tracing the left (cooling) branch, eventually forming a closed loop between two transition-end points $\mathrm{T}_{\mathrm{S}}$ and $\mathrm{T}_{\mathrm{M}}$. As can be seen in Figs. $1-4$, resistivity below the transition, in the S-phase, is about 3 orders of magnitude higher than that above the transition, in M-phase. The hysteresis width in the middle of this $\log (\mathrm{R})$ vs. T plot is about $15^{\circ} \mathrm{C}$, while the total width of a hysteretic region is $\mathrm{T}_{\mathrm{M}}-\mathrm{T}_{\mathrm{S}} \approx 50{ }^{\circ} \mathrm{C}$.

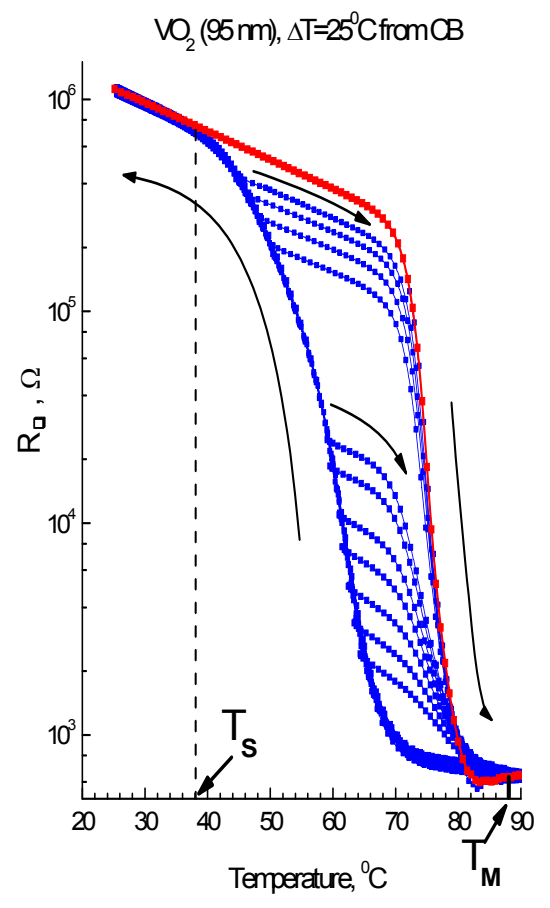

(a)

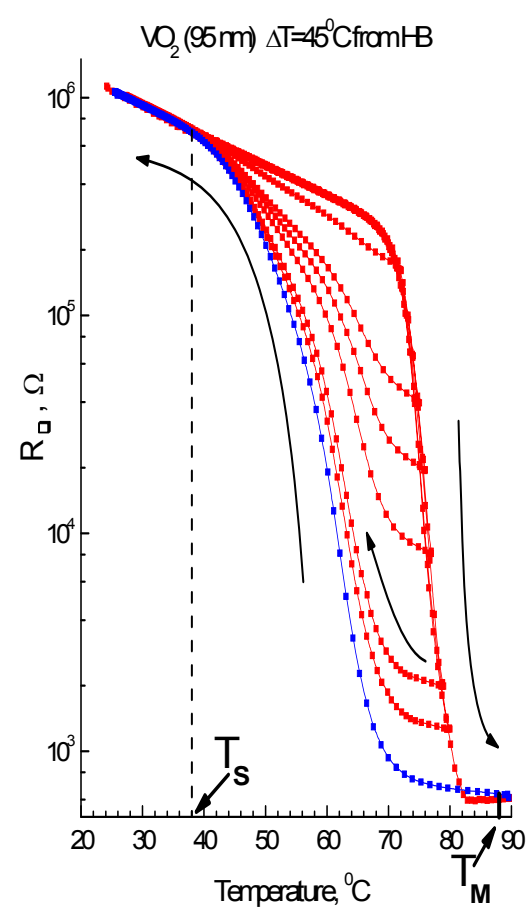

(b)

Figure 1. Major hysteresis loop of $\mathrm{VO}_{2}$ film with a number of minor loops initiated from various temperatures $T_{0}$ on (a) the cooling branch, $T_{0} \rightarrow T_{0}+\Delta T \rightarrow T_{0}, \Delta T=25$ ${ }^{\circ} \mathrm{C}$, and (b) the heating branch, $\mathrm{T}_{0} \rightarrow \mathrm{T}_{0}-\Delta \mathrm{T} \rightarrow \mathrm{T}_{0}, \Delta \mathrm{T}=45^{\circ} \mathrm{C}$. 
For a variety of reasons, of the many proposals to use this transition in applications almost none materialized. One commercial and military application in which the use of $\mathrm{VO}_{2}$ was initially envisioned is IR visualization (night vision) based on resistive microbolometers. However, technology eventually settled on a $\mathrm{VO}_{2}-\mathrm{S}$ poor cousin, a non-transitioning mixed oxide $\mathrm{VO}_{\mathrm{x}}$, mainly to avoid hysteresis, which greatly complicates bolometer operation, as was mentioned in the introduction and will be discussed in more detail below.

The major loop encompasses all the points on the (T, R) plane which can serve as starting points of minor hysteresis loops. In particular, minor loops can be initiated at any point along the outer lines of the major loop. In Fig. 1 we show several minor loops which were traced by reversing the temperature change at various points along the major loop: in Fig. 1a, minor loops are starting along the points of the cooling branch (CB); in Fig. 1b: along the points of the heating branch (HB). The loops shown in Fig. 1 cover wide temperature ranges of $25^{\circ} \mathrm{C}$ in $1 \mathrm{a}$ and $45^{\circ} \mathrm{C}$ in 1 b. A number of minor loops traced over a more narrow temperature range of 10 ${ }^{\circ} \mathrm{C}$ are shown in Fig. 2.

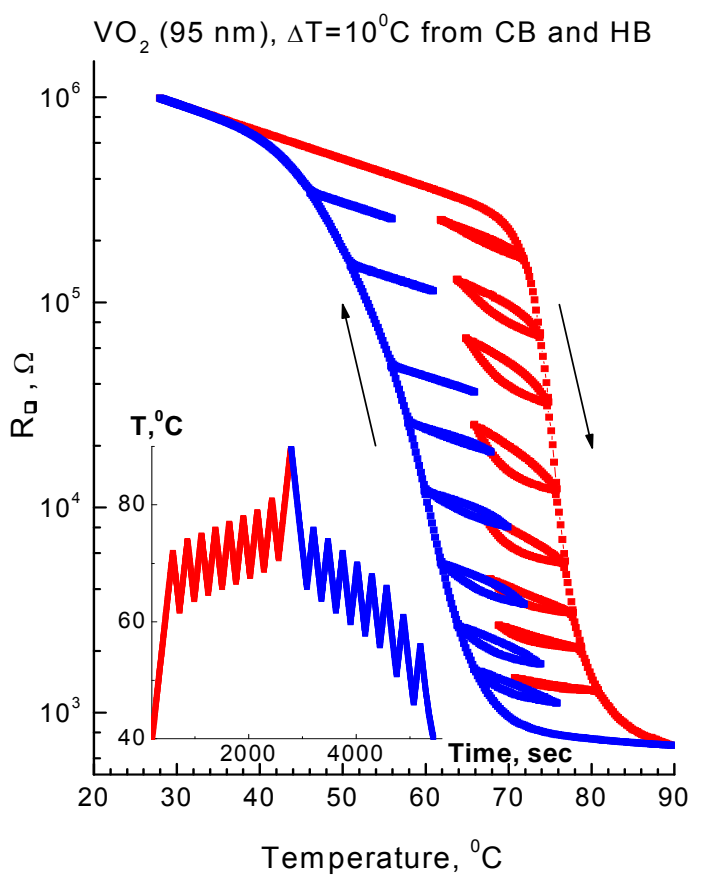

Figure 2. Minor loops with $\Delta \mathrm{T}=10^{\circ} \mathrm{C}$; the inset shows the way in which temperature was changed in this measurement.

3. How hysteresis causes problems in bolometric readout; forward and backward excursions.

A bolometer reacts to a temperature change $\Delta \mathrm{T}$ by changing its resistance by $\Delta \mathrm{R}$. The larger is $\Delta \mathrm{R}$ for a given $\Delta \mathrm{T}$, the greater is the sensitivity. At the same time, 
very large resistance $\mathrm{R}$ is detrimental because of bolometric sensor Joule heating during readout, difficulty of matching to the electronic readout circuit and higher noise, both Johnson and $1 / \mathrm{f}^{[1]}$. Thus a commonly-used measure of the bolometric material's sensitivity is the logarithmic derivative (1/R) $\Delta \mathrm{R} / \Delta \mathrm{T}$ called temperature coefficient of resistance, or TCR. In other words, it is not the high $\Delta \mathrm{R}$ but high $\Delta \mathrm{R} / \mathrm{R}$ which is desired.

The steep semiconductor to metal transition seem to promise great sensitivity (high TCR), comparable to the sensitivity which can be achieved in a transition-edge superconducting sensor, which provided the original incentive to employ such a transition in $\mathrm{VO}_{2}{ }^{[4]}$. Why then was it not used? The main reason it was not used is because of hysteresis.

Let us look in more detail on how hysteresis causes problems. To be specific, let us consider a bolometer positioned at a working point $\left(\mathrm{T}_{0}, \mathrm{R}_{0}\right)$ on a steep heating branch (HB) (it will require a temperature controller to keep it at $T_{0}$ above room temperature) and let it experience an influx of energy (this could be an IR signal) which momentarily heats it up, increasing its temperature by $\Delta \mathrm{T}$ and decreasing its resistance by a large $\Delta R$ (Fig. 3).

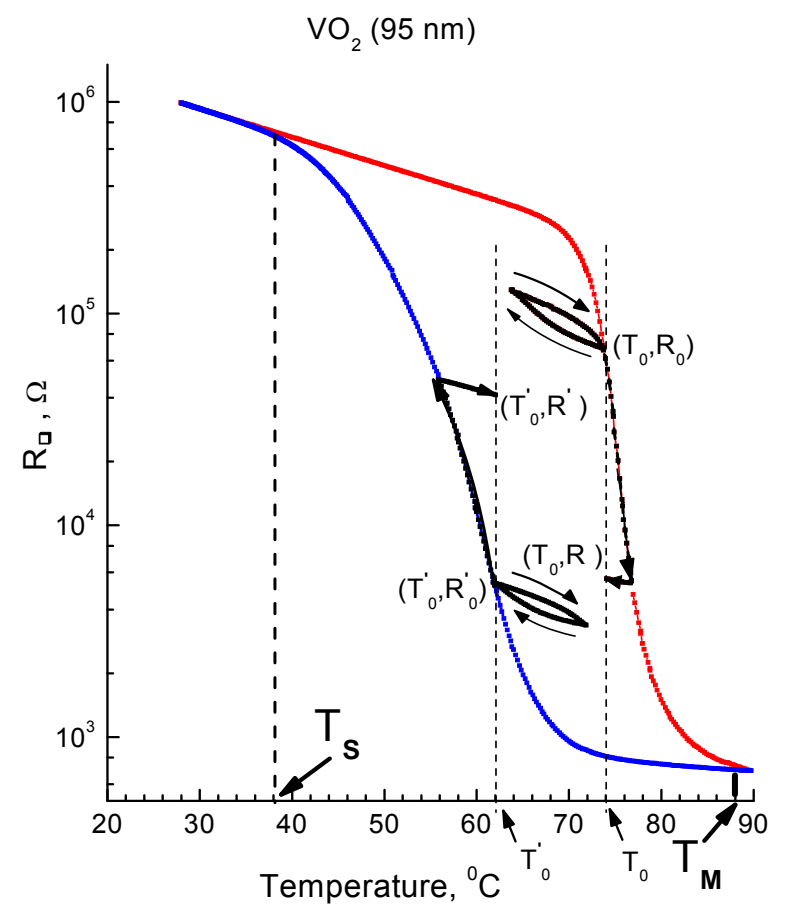

Figure 3. Forward excursions originating from point $\left(T_{0}, R_{0}\right)$ on a $H B$ and from point $\left(T_{0}^{\prime}, R_{0}^{\prime}\right)$ on a $C B$, tracing open curves ending at $\left(T_{0}, R\right)$ on a $H B$ and at $\left(T_{0}^{\prime}, R^{\prime}\right)$ on a $C B$. The backward excursions from $\left(T_{0}, R_{0}\right)$ and $\left(T_{0}^{\prime}, R_{0}^{\prime}\right)$ return to the points of their origin following closed minor loops. Note that this figure is not a schematic drawing; it shows measured loops and parts of loops. 
Once the heat input has been removed (which happens in resistive bolometers used in IR visualization at least 30 times per second), the bolometer returns back to the temperature $\mathrm{T}_{0}$; however, because of the hysteresis, the $(\mathrm{T}, \mathrm{R})$ path on the way back differs from the path in the forward direction: the resistance will not come back to $\mathrm{R}_{0}$, but instead will move along the part of the minor loop to a point $\left(\mathrm{T}_{0}, \mathrm{R}\right)$ as indicated in Fig. 3. If a bolometer will now experience a subsequent, second heat pulse, the result will be very different. This can not be tolerated in a bolometric sensor subject to a train of pulses. In order to avoid this problem the bolometer should be reset after each pulse, which can only be done by going all the way out of the hysteresis loop, reaching temperatures either below $T_{S}$ or above $T_{M}$, as can be seen in Fig. 1. While such resetting can be done, it requires large temperature excursions (we will call a round-trip temperature change $\mathrm{T}_{0} \rightarrow \mathrm{T}_{0} \pm \Delta \mathrm{T} \rightarrow \mathrm{T}_{0}$ an excursion) followed each time by temperature stabilization at the working point $\mathrm{T}_{0}$. With bolometers receiving signals with frequency of $30-60 \mathrm{~Hz}$ (video rates) this is not practical. The problem with the different return path we just outlined has been described in the literature ${ }^{[5]}$, and the readout problems caused by hysteresis were acknowledged $^{[6]}$ as the major contributing factor in killing the original proposal ${ }^{[4]}$ of using high TCR found in a $\mathrm{VO}_{2}$ transition.

A word about terminology: as was said, we call a round trip $T_{0} \rightarrow T_{0} \pm \Delta T \rightarrow T_{0}$ an excursion. We consider excursion length $\Delta \mathrm{T}$ a positive quantity, and show the plus or minus sign in front of it explicitly. If temperature of the initial part of an excursion changes in the same direction as the major loop progression, we call it a forward excursion; if it starts in the opposite direction, it is a backward excursion. Thus in Fig. 3 on the HB the excursion from $\left(T_{0}, R_{0}\right)$ to $\left(T_{0}, R\right)$ is a forward one; the other one, forming a closed loop, is a backward one. We could discuss similar processes originating from a point $\left(\mathrm{T}_{0}^{\prime}, \mathrm{R}_{0}^{\prime}\right)$ on a $\mathrm{CB}$ (they are also shown in Fig. 3 ), keeping in mind that on a $\mathrm{CB}$ decreasing temperature corresponds to the forward direction, and increasing temperature to the backward direction.

Because of the hysteresis, forward and backward excursions produce dramatically different results. While a forward excursion $\mathrm{T}_{0} \rightarrow \mathrm{T}_{0}+\Delta \mathrm{T} \rightarrow \mathrm{T}_{0}$ on a HB traces an open curve from $\left(T_{0}, R_{0}\right)$ to $\left(T_{0}, R\right)$, a backward excursion $T_{0} \rightarrow T_{0}-\Delta T \rightarrow T_{0}$ produces a closed minor loop, which has a much smaller slope near $\left(\mathrm{T}_{0}, \mathrm{R}_{0}\right)$ than the steep slope on the major loop, this slope increasing for larger $\Delta \mathrm{T}$, as can be seen in Fig. 3 and as can be inferred from Fig. 1. This will introduce signal nonlinearity in $\log (\mathrm{R})$ vs. $\mathrm{T}$, involving changing TCR which depends on the value of $\Delta \mathrm{T}$. Similar processes take place on a CB (Fig. 3).

To summarize, hysteresis causes the following two distinct types of problems:

- Forward excursions produce open (T,R) curves ("memory" effects), which are un-acceptable in a bolometer operating in a regime of repeating, multiple measurements.

- Backward excursions produce closed loops, so that bolometer re-setting is not required. However, the double-valued nature of such a minor loop makes readout ambiguous, and variable TCR found in minor loops is unacceptable, or at least detrimental, producing non-linearity in the 
bolometric response, with small $\Delta \mathrm{T}$ corresponding to smaller TCR, larger $\Delta \mathrm{T}$ to larger $\mathrm{TCR}$.

\section{Non-hysteretic branches (NHB) in resistivity}

Let us examine Fig.2 in more detail. We see that most of the minor loops in Fig. 2 are hysteretic, but we also notice that some of them are rather flat, single-valued, particularly those near the major loop ends $\mathrm{T}_{\mathrm{S}}$ and $\mathrm{T}_{\mathrm{M}}$. In studying minor loops with progressively smaller excursions $\Delta \mathrm{T}$ we discovered that for sufficiently small $\Delta \mathrm{T}$ minor loops flattened out, degenerating into what we call non-hysteretic branches (NHB's). Although some minor loops become flat with $\Delta \mathrm{T}=10^{\circ} \mathrm{C}$ (Fig. 2), all of them become flat at or below $\Delta \mathrm{T}_{\mathrm{NHB}}=4^{\circ} \mathrm{C}-5^{\circ} \mathrm{C}$, as can be seen in Fig.4:

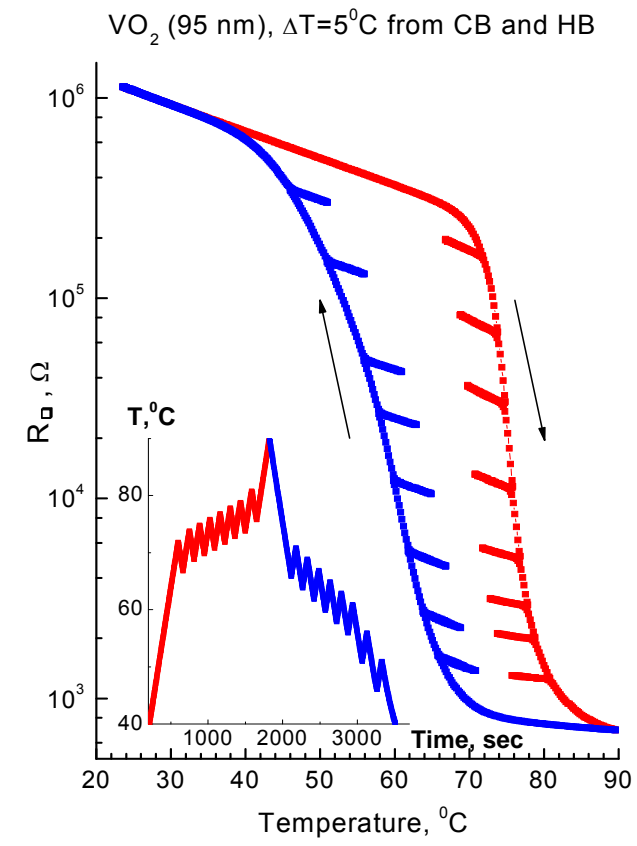

Figure 4. Minor loops with $\Delta T=5{ }^{\circ} \mathrm{C}$; the inset shows the way in which temperature was changed in this measurement.

A NHB can be initiated from any point on the major loop, either on a HB or on a CB; NHB-s are linear in $\log (\mathrm{R})$ vs. $\mathrm{T}$ with negative slopes (semiconducting TCRs). Two representative NHB-s measured in round-trip excursions are plotted on $\ln (\mathrm{R})$ vs. T scales in Fig. 5a, b. 


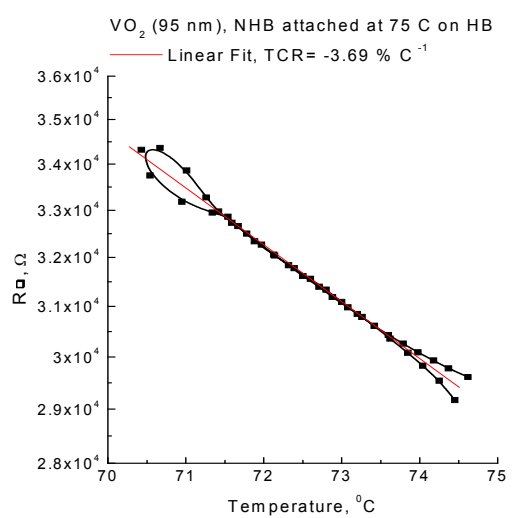

(a)

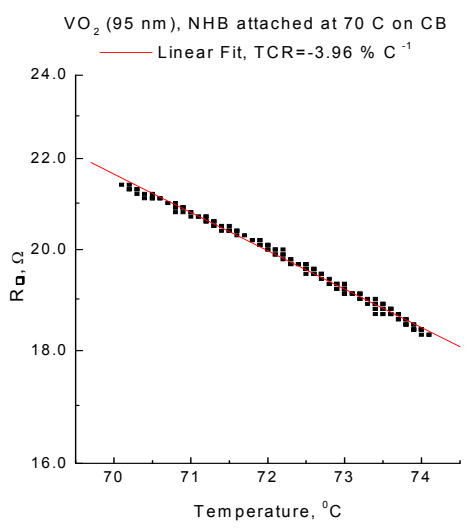

(b)

Figure 5. (a) NHB attached to the HB, with $\mathrm{R}_{\square} \approx 31 \mathrm{k} \Omega$; TCR $=-3.69 \%{ }^{\circ} \mathrm{C}^{-1}$; (b) NHB attached to the $\mathrm{CB}$, with $\mathrm{R}_{\square} \approx 20 \Omega$; despite this low resistance, $T C R=-3.96$ $\%{ }^{\circ} \mathrm{C}^{-1}$

NHB-s are single valued to the precision of our measurements, except for the temperature intervals near the attachment point $T_{0}$ and the turning point $T_{0} \pm \Delta T$, where they sometimes appear double-valued, exhibiting a small loop and a fork, as seen in Fig. 5a. These features depend on the rate of temperature sweep; they are probably instrumental effects resulting from a lag between film surface and thermometer temperatures ${ }^{[1]}$.

TCR-s of various NHB-s from Fig. 4 are plotted vs. $T_{0}$ in Fig. 6 for both HB and CB.

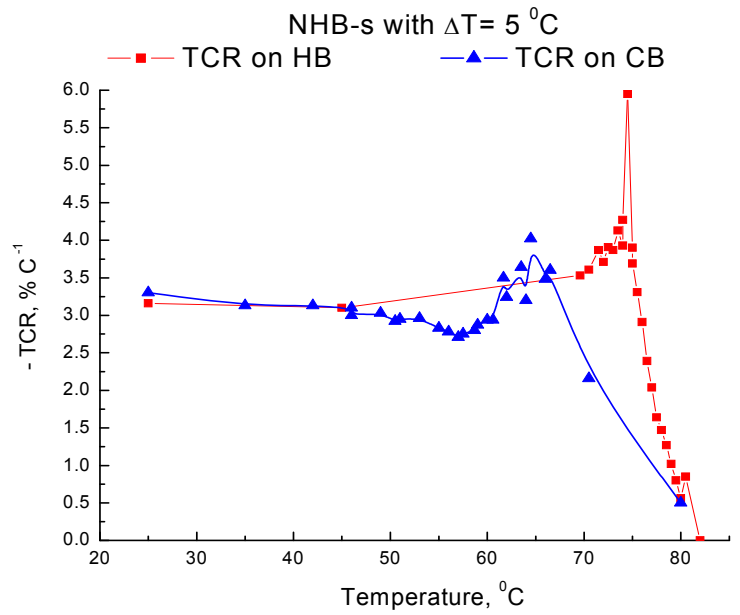

Figure 6. Absolute values of TCR plotted versus $T_{0}$ for various NHB-s around the major loop. 
The S-phase (TCR) $)_{\mathrm{S}}$ at $25 \mathrm{C}$ is $3.3 \%{ }^{\circ} \mathrm{C}^{-1}$, which is considerably higher than a typical TCR $\approx 2 \%{ }^{\circ} \mathrm{C}^{-1}$ at $25{ }^{\circ} \mathrm{C}$ found in the literature on $\mathrm{VO}_{\mathrm{x}}{ }^{[1,6]}$. In an intrinsic semiconductor $\mathrm{E}_{\mathrm{g}}=2(|\mathrm{TCR}|) \mathrm{k}_{\mathrm{B}} \mathrm{T}^{2}{ }^{[1]}$, and substituting $|\mathrm{TCR}|=0.033$ and $\mathrm{T}=25$ $+273.1=298.1 \mathrm{~K}$, we obtain $\mathrm{E}_{\mathrm{g}}=0.51 \mathrm{eV}$, in fair agreement with the bandgap value in $\mathrm{VO}_{2}$ single crystals ${ }^{[3]}$.

As we see in Fig. 6, NHB-s logarithmic slopes are peaking at $65{ }^{\circ} \mathrm{C}$ on the $\mathrm{CB}$ and $75{ }^{\circ} \mathrm{C}$ on the $\mathrm{HB}$, with maximum values of $4 \%{ }^{\circ} \mathrm{C}^{-1}$ and $6 \%{ }^{\circ} \mathrm{C}^{-1}$, decreasing sharply above the peaks. We note that the highest TCR-s on the sides of the major loop are found at the same temperatures.

\section{Theoretical model}

We will present a theoretical model which provides a qualitative explanation of the observed behavior and, moreover, has predictive power. Specific predictions made with our model will be put to a test by comparing them to experiment in the next section.

The hysteretic region in $\mathrm{VO}_{2}$ is a mixed state consisting of both the S-phase and Mphase regions, or domains. Each such region located in a film around a point with spatial coordinates $(\mathrm{x}, \mathrm{y})$ transitions into the other phase at its own temperature $\mathrm{T}_{\mathrm{C}}$ $(\mathrm{x}, \mathrm{y})$, the variation in $\mathrm{T}_{\mathrm{C}}$ arising from non-uniformities of composition, variations in the local strain, etc. Thus in a macroscopic sample $\mathrm{T}_{\mathrm{C}}(\mathrm{x}, \mathrm{y})$ is quasi-continuously distributed. We assume that the local transition within a domain is sharp; further, we assume that a uniform isolated domain would transition without a hysteresis; in this we differ from much of the $\mathrm{VO}_{2}$ hysteresis literature ${ }^{[7]}$ in which it is usually assumed that each domain, in addition to $\mathrm{T}_{\mathrm{C}}$, has its own coercive temperature and a rectangular hysteresis loop. In contrast, we do not see a need for postulating such intrinsic hysteresis in isolated domains. We note that single crystals have very small hysteresis ${ }^{[3]}$; it seems natural to extrapolate this to the case of an ideallyuniform microscopic (or nanoscopic) region which would have zero hysteresis. In our picture hysteresis is the result of interaction between different phases in a multi-domain macroscopic sample, as will be detailed below.

At a given temperature $\mathrm{T}$ inside the major hysteretic loop, some parts of the film have $\mathrm{T}_{\mathrm{C}}(\mathrm{x}, \mathrm{y})<\mathrm{T}$ and some $\mathrm{T}_{\mathrm{C}}(\mathrm{x}, \mathrm{y})>\mathrm{T}$. In the first approximation, the boundary wall between the $S$ and $M$ phases is determined by the condition $T_{C}(x, y)=T$. In this approximation, the wall is highly irregular and its ruggedness corresponds to the scale at which one can define the local $\mathrm{T}_{\mathrm{C}}(\mathrm{x}, \mathrm{y})$, i.e. to the characteristic length scale of the nanoscopic phase domains. On closer inspection, however, we need a refinement that takes into account the boundary energy, associated with the phase domain wall itself. The boundary energy is positive and to minimize its contribution to the free energy the domain walls are relatively smooth.

Let us examine the process of boundary motion. For concreteness, let us consider the heating branch. Below the percolation transition, $\mathrm{M}$ phase resembles lakes in 
the $\mathrm{S}$ phase mainland. With raising temperature the area of the $\mathrm{M}$ phase increases, lakes grow in size. When a boundary of a given lake is far from the other lakes, infinitesimal $\pm \mathrm{dT}$ changes boundary length by infinitesimal amount $\pm \mathrm{dL}$, and the lake area by $\pm \mathrm{dA}_{\mathrm{M}}$. In other words, when the lakes are sufficiently separated, we envision a continuous, reversible, hysteresis-free process of $\mathrm{M} \leftrightarrow \mathrm{S}$ area redistribution, with neighboring configurations differing microscopically, what we call area breathing.

Let us now look at the formation of a link between two neighboring regions, which is the elementary step in the topological evolution of a global percolation picture. Let us focus on two metallic lakes that are about to merge. Since the boundary is smooth, at some temperature the distance between the lakes becomes smaller than the radius of curvature of either lake at the point they will eventually touch. Therefore, at some $\mathrm{T}=\mathrm{T}_{\text {cr }}$ the following two configurations will have equal energies: one comprising two disconnected $\mathrm{M}$ phase lakes that are near touching, but not quite, and the other with a finite link formed, Figs. 7a and $7 \mathrm{~b}$ respectively.

(a)

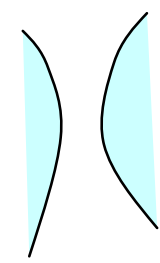

(c)

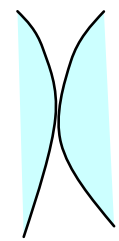

(b)

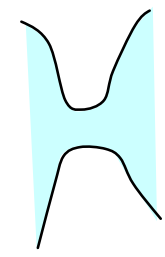

(d)

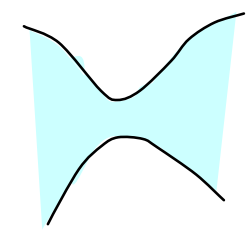

Figure 7. Semiconductor-metal boundary; metallic phase is shown shaded. Top row (a), (b) corresponds to temperature $T_{1}$ and the bottom row (c), (d) to temperature $T_{2}>T_{1}$.

Both configurations are characterized by equal boundary lengths and therefore have equal free energy. In the thermodynamic sense one could call the $T_{c r}$ the critical temperature for the link formation, if we could wait long enough. The actual transition forming a local link, however, does not occur at that temperature because of an immense kinetic barrier between these two macroscopically different configurations. The transition occurs at a higher $\mathrm{T}_{0}=\mathrm{T}+\Delta \mathrm{T}^{*}$ when it is actually forced, i.e. when the two phases touch at a point. Here $\Delta \mathrm{T}^{*}$ is the coercive temperature. As can be seen, in our picture coercive temperature arises as a result of having a boundary between different phases; it does not pre-exist intrinsically within each domain. We associate the steep slopes of the major loop with the quasi-continuous formation of such links, i.e. with local topological changes, specifically with the merger of metallic lakes on the HB and semiconductor lakes 
on the $\mathrm{CB}$. Near $\mathrm{T}_{\mathrm{S}}$ and $\mathrm{T}_{\mathrm{M}}$ the global map consists of widely-separated $\mathrm{M}$ and $\mathrm{S}$ lakes, respectively. In these regions we expect to see non-hysteretic behavior; indeed, looking at Fig. 3 we see that $\Delta \mathrm{T}=10^{\circ} \mathrm{C}$ NHB-s are largely non-hysteretic within $\approx 20^{\circ} \mathrm{C}$ from $\mathrm{T}_{\mathrm{S}}$ and $\approx 15^{\circ} \mathrm{C}$ from $\mathrm{T}_{\mathrm{M}}$.

Consider now a small excursion backwards from $\mathrm{T}_{0}$ on the $\mathrm{HB}$. As the temperature decreases, some of the M-phase recedes and the S-phase grows, changing the geometry of the global two-phase map. However, topologically, the last formed Mlink does not disappear immediately for the same kinetic reason. One has two $\mathrm{S}$ regions that need to touch in order to wipe out the M-link. It takes a backward excursion of amplitude $\Delta \mathrm{T}^{*}$ to establish an S-link and thus disconnect the last $\mathrm{M}$ link. So long as we are within $\Delta \mathrm{T}^{*}$, i.e. stay on the same NHB, the area of $\mathrm{S}$ and $\mathrm{M}$ domains changes continuously, but the topology is stable and no new links are formed. Within the range of that stable or frozen topology, $\Delta \mathrm{T}^{*}=\Delta \mathrm{T}_{\mathrm{NHB}}$, the resistivity of NHB will be single-valued and its T-dependence will be controlled by the percolating semiconductor phase. This explains why NHB-s have semiconducting slopes.

We introduced here a notion of frozen topology, which describes a global twophase map with stable connecting links between regions of the same phase, while geometrical shape of the two phases is allowed to change, or breathe. One can also envision NHB-s in which breathing stops altogether, no switching between phases taking place, keeping the geometrical outlines fixed, this being the case of frozen geometry. Clearly frozen geometry implies frozen topology, but not the other way around. We will see below that both conditions actually exist and can be observed in a transitioning $\mathrm{VO}_{2}$.

We can further address the observed phenomenon of TCR enhancement in some of the NHB-s, where TCR-s exceed the S-phase value (Fig.6). We note that for all NHB-s, on both branches of the major loop, higher $T$ implies increased fraction of M-phase, even if no new links are formed. This smooth change in geometry will produce additional T-dependence adding to the semiconductor slope within a NHB, i.e. higher TCR. This effect will be stronger in the temperature intervals where this smooth change in geometry is more active, and it will cease to exist in the regions of frozen geometry, where NHB slope will be determined only by the percolating S-phase. We will refer to this effect vis-à-vis optical data below.

Theoretical models which explain the data are good, but those which correctly predict new behavior are better. What can we predict based on our model?

We recall that hysteresis takes place in all physical properties which change in the phase transition. In addition to resistivity, optical constants, such as refractive index $\mathrm{n}$, change in the $\mathrm{VO}_{2}$ transition. In a small uniform domain $\mathrm{n}$ changes abruptly at $T_{C}$, from $n_{S}$ to $n_{M}$; in a macroscopic film there is a distribution of $\mathrm{T}_{\mathrm{C}}(\mathrm{x}, \mathrm{y})$ and therefore, in a temperature range of co-existing $\mathrm{S}$ and $\mathrm{M}$ phases, $\mathrm{T}_{\mathrm{S}}<\mathrm{T}$ $<\mathrm{T}_{\mathrm{M}}$, there will be some $\mathrm{S}$ domains with $\mathrm{n}_{\mathrm{S}}$ and some $\mathrm{M}$ domains with $\mathrm{n}_{\mathrm{M}}$. As phases transform with changing $\mathrm{T}$, there will be a temperature dependent hysteretic $\mathrm{n}(\mathrm{T})$ in a macroscopic film, much like previously discussed $\mathrm{R}(\mathrm{T})$. Indeed, optical 
properties exhibit hysteretic transition as a function of temperature. Among them, optical reflectivity is probably the easiest optical quantity to measure; the details of how optical reflectivity behaves in a phase transition in a film with thickness comparable with the wavelength of light have to do not only with $n(T)$ but also with thin-film interference; this was discussed in Ref. 8 (and in references therein), and we will not repeat the explanation here. The result is that, when $\mathrm{T}$ increases from $25 \mathrm{C}$ to $100 \mathrm{C}$ and decreases back to $25 \mathrm{C}$, optical reflectivity $\mathrm{R}_{\lambda}(\mathrm{T})$ at a fixed wavelength $\lambda$ traces a hysteresis loop between certain values $R_{\lambda S}$ and $R_{\lambda M}$, these values being determined by a number of factors, including the choice of $\lambda$, film thickness, $\mathrm{n}_{\mathrm{S}}$ and $\mathrm{n}_{\mathrm{M}}$. We can further expect that backward excursions from the points on the major optical loop will produce optical NHB-s, for the same reason they exist in $\mathrm{R}(\mathrm{T})$, and indeed we observed them, as will be seen below.

Based on our theoretical model, what specific behavior can we predict in optical reflectivity vs. T? Reflectivity is measured at wavelengths exceeding the nanodomain scale. It is largely determined by thin-film interference, with two-phase material between the film surfaces (in the Fabry-Perot resonator) having the average value of $n$, the latter depending on the volume fraction of $\mathrm{S}$ and $\mathrm{M}$ phases (volume fraction of $n_{S}$ and $n_{M}$ ), which in a thin film becomes the area fraction of the two phases. In contrast, resistivity depends on the connectivity (links) of the percolating phase. Let $A_{S}$ and $A_{M}$ be the areas of S-phase and M-phase in a twodimensional sample (thin film), so that the total sample area is $A=A_{S}+A_{M}$. Clearly, as A does not depend on $\mathrm{T}, \mathrm{dA}_{\mathrm{M}} / \mathrm{dT}=-\mathrm{dA}_{\mathrm{S}} / \mathrm{dT}$, i.e. the area of one phase grows at the expense of the other. The optical slope $\mathrm{dR}_{\lambda} / \mathrm{dT}$ observed in optical NHB must be proportional to this area re-distribution slope, with the maximum in $\mathrm{dR}_{\lambda} / \mathrm{dT}$ reflecting the maximum in $\mathrm{dA}_{\mathrm{M}} / \mathrm{dT}$. According to our explanation of TCR enhancement over the S-phase value, the highest TCR should be found at the point which has the highest rate of area re-distribution $\mathrm{dA}_{\mathrm{M}} / \mathrm{dT}=-\mathrm{dA}_{\mathrm{S}} / \mathrm{dT}$, and, therefore, at the point which corresponds to the maximum optical NHB slope $\mathrm{dR}_{\lambda} / \mathrm{dT}$. Likewise, zero rates of area re-distribution (frozen geometry) corresponding to $\mathrm{dR}_{\lambda} / \mathrm{dT}=0$, should correspond to un-enhanced S-phase TCR. In the next section we will have a chance to verify these specific predictions.

The percolation picture also helps to understand why $\mathrm{dR}_{\lambda} / \mathrm{dT}$ will exhibit such a maximum in the first place. With changing temperature, the boundary moves, each section of the boundary line advancing in the direction normal to this line at any given temperature. It is clear that the highest rate of change of the area of each phase will therefore occur when the boundary is the longest, i.e. at the percolation transition. If our picture is correct, then the observed peak in TCR vs. $\mathrm{T}_{0}$ in Fig. 6 occurs right at the percolation transition, allowing its detection. Finally, we see in Fig.6 that above the peak, i.e. above the percolation transition, TCR-s are quickly decreasing. Indeed, if the M-phase percolates, it is shorting out the S-phase, and such decrease is to be expected. 


\section{NHB-s in optical reflectivity: testing the theoretical model}

In order to test some of the predictions of our model, simultaneously with the resistive measurements presented above in Figs. 2 and 4, we measured reflectivity at a fixed wavelength $\lambda, R_{\lambda}(T)$. Optical signal was taken from the space between the voltage probes used in 4-probe resistivity measurement. In Fig. 8 we show major loop and minor hysteresis loops with $\Delta \mathrm{T}=10^{\circ} \mathrm{C}$ in optical reflectivity $\mathrm{R}_{\lambda}(\mathrm{T})$ (i.e. this is an optical analog of Fig. 2).

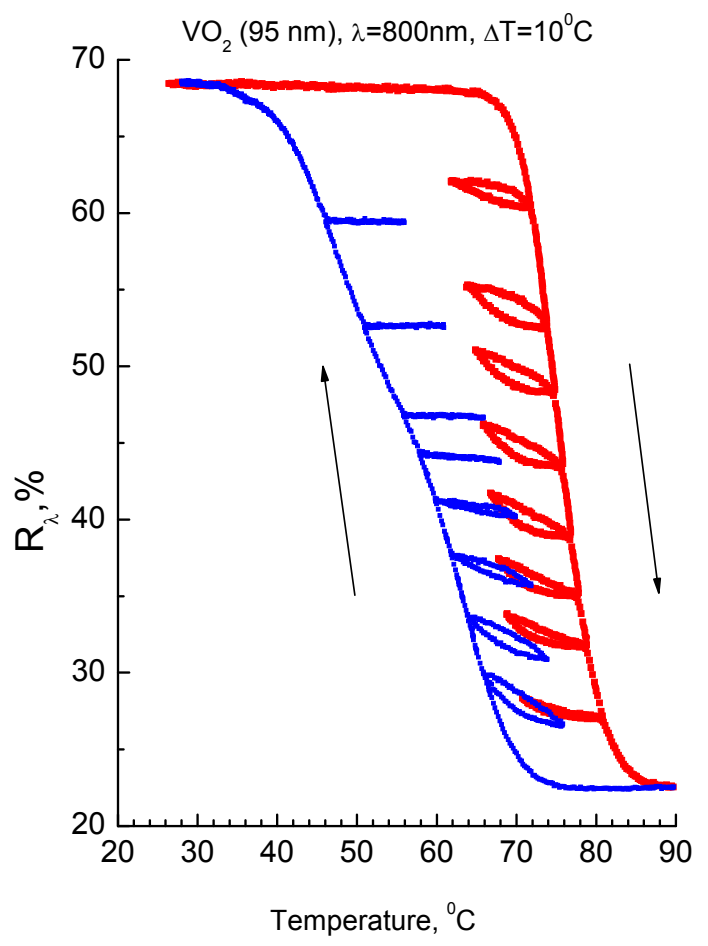

Figure 8. Major hysteresis loops in optical reflectivity with minor loops, excursion length $\Delta \mathrm{T}=10^{\circ} \mathrm{C}$, measured at $\lambda=800 \mathrm{~nm}$.

In Fig. 8 we see minor loops, some of them degenerating into optical NHBs, just like in resistivity in Fig. 2. Additionally we see that some minor loops are Tdependent, while others are not, and that T-dependent ones tend to be doublevalued loops, while T-independent ones are degenerating into NHB-s. In Fig. 9 we plot data for the same sample with short $5{ }^{\circ} \mathrm{C}$ excursions (i.e. this figure is an optical analog of Fig. 4). 


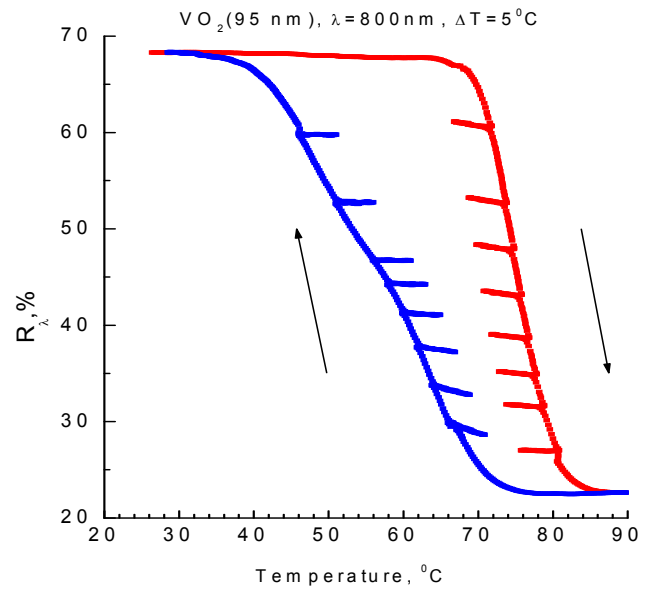

Figure 9. Data as in Fig. 8, but with $\Delta \mathrm{T}=5^{\circ} \mathrm{C}$.

We see that all minor loops degenerated into NHB-s, in complete analogy to Fig. 4, and according to our expectation expressed in sec. 5. Some of these NHB-s, those closer to the center of the major loop, are T-dependent, the others, those closer to the loop limits, are not. The T-dependent ones correspond to hysteretic minor loops in Fig. 8, while T-independent NHB-s correlate with non-hysteretic behavior in Fig. 8.

Optical slopes $\mathrm{dR}_{\lambda} / \mathrm{dT}$ of NHB-s from Fig.9 are plotted alongside TCR-s from Fig.4 in the combined Fig.10, for both HB and CB.

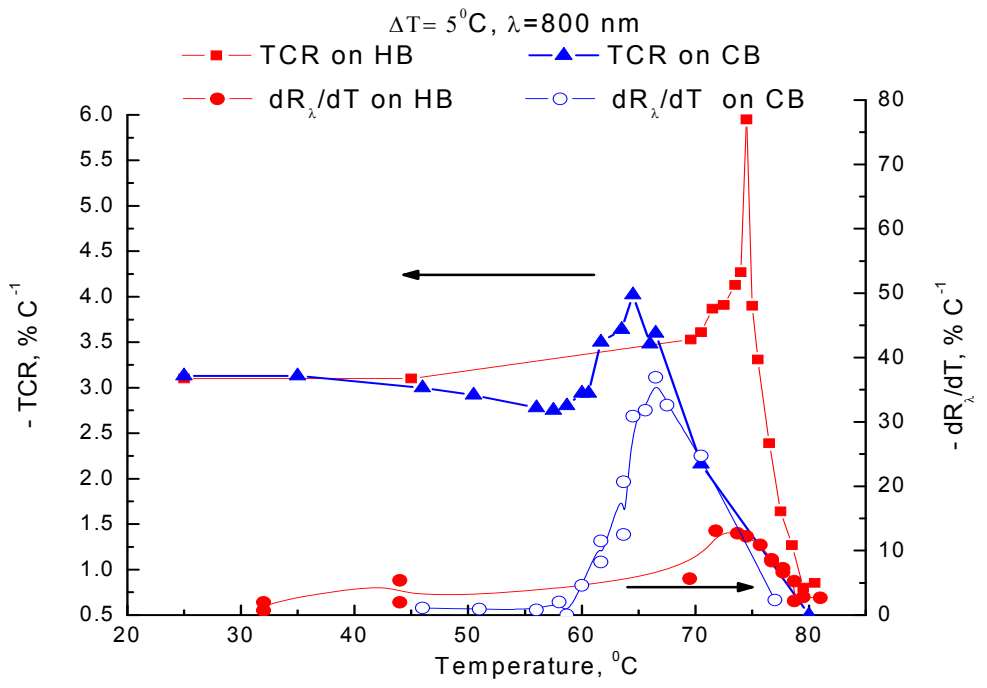

Figure 10. TCR-s and $d R_{\lambda} / d T$-s from various NHB-s around the major loop plotted on one graph, exhibiting expected correlations. 
As can be seen, the specific predictions of our model are indeed proven true: the peaks in $\mathrm{dR}_{\lambda} / \mathrm{dT}$ on both branches almost precisely coincide with the peaks in TCR, and the region in which $\mathrm{dR}_{\lambda} / \mathrm{dT} \approx 0$ corresponds to $\mathrm{TCR} \approx(\mathrm{TCR})_{\mathrm{S}}$. Further, if $\mathrm{dR}_{\lambda} / \mathrm{dT} \approx 0$ signifies frozen geometry, it is natural that in the absence of $\mathrm{S} \leftrightarrow \mathrm{M}$ transitions minor loops degenerate into NHB-s more readily, as is observed when we examine and compare Figs. 8 and 9 . The small $(\approx 2$ degrees) misalignment of $\mathrm{dR}_{\lambda} / \mathrm{dT}$ and TCR peaks which can be seen in Fig. 10 can be ascribed to the fact that the factors affecting the overall shape of the major optical loop, such as interference effects ${ }^{[8]}$, affect, at least to a degree, NHB slopes as well. Likewise, a relatively small deviation of TCR-s in the $\mathrm{dR}_{\lambda} / \mathrm{dT} \approx 0$ region from the S-phase value - the observed shallow TCR minimum on the $\mathrm{CB}$ - can be ascribed to secondary causes. We do not think that these details disprove or diminish the impressive overall agreement of the main features predicted by our model.

As was said above, the overall shape of the optical hysteresis and the range between pure-phase reflectivity values of $R_{\lambda S}$ and $R_{\lambda M}$ can be changed by making measurements at different wavelengths $\lambda$. We measured $\mathrm{R}_{\lambda}(\mathrm{T})$ at two different $\lambda$; the measurement at $\lambda=800 \mathrm{~nm}$ is shown in Figs. 8 and 9, and we also measured it at $\lambda=503 \mathrm{~nm}$. The measurement at $503 \mathrm{~nm}$ produces an optical loop which looks entirely different than the one shown in Figs.8 and 9. Yet we observed $d_{R_{\lambda}} / d T$ having the same peaks as seen in Fig. 10, these peaks correlating with TCR peaks, which strongly confirm that we are indeed observing intrinsic $\mathrm{S} \leftrightarrow \mathrm{M}$ area redistribution and not the effects of interference, etc.

Summing up, we proposed a theoretical picture, expressed in sec. 5 in qualitative terms, without detailed mathematical modeling, which would be rather involved (perhaps a task for the future). We describe hysteresis in the framework of a percolation transition, as arising from interaction of $S$ and $M$ phases, specifically ascribing strong hysteretic effects to link formation between the two phases. This model corresponds to a number of observed features in both resistive and optical data. The most impressive agreement is achieved in the explanation of a subtle effect of TCR enhancement over the S-phase value in a range of NHB-s where optical slopes correlate with TCR-s, leaving no doubt that we understand the reason for TCR enhancement correctly.

\section{Resistive microbolometers in NHB regime}

A detailed discussion of the NHB method in the context of IR visualization with resistive microbolometers (the Uncooled Focal Plane Array or UFPA technology) is given in Ref. 1. Here we only give a brief summary. Good quality, single phase $\mathrm{VO}_{2}$ films will replace mixed oxide $\mathrm{VO}_{\mathrm{x}}$ as sensor material in pixilated bolometric array. Despite using $\mathrm{VO}_{2}$, hysteresis is eliminated when a sensor array operates within a NHB. The NHB will be chosen on the basis of its desired resistance, which can be adjusted in a wide range in order to be matched to the readout circuit amplifier, and to be low enough to minimize noise and Joule heating. The resistance will be 2-3 orders of magnitude smaller than the unacceptably-high $\mathrm{VO}_{2}$ 
resistance at $25{ }^{\circ} \mathrm{C}$, while maintaining semiconducting TCR. We have seen NHB-s with resistance as low as $20 \Omega$ having TCR $\approx-4 \%{ }^{\circ} \mathrm{C}^{-1}$. NHB will be also chosen to maximize TCR, which, as we have seen, varies between different NHB-s around the major loop, peaking at the percolation transition, with values as high as $6 \%{ }^{\circ} \mathrm{C}^{-1}$. The operating temperature $\mathrm{T}_{\mathrm{OP}}$ (i.e. the temperature at which the sensor array is stabilized awaiting the projected IR signal) will be chosen within a NHB, either near one of the ends or in the middle of the available range (total NHB width) $\Delta \mathrm{T}_{\mathrm{NHB}}=4-5{ }^{\circ} \mathrm{C}$. Because of the hysteresis, the process of reaching $\mathrm{T}_{\mathrm{OP}}$ starting from room temperature requires performing specific heating and cooling steps. Specifically, positioning an array at $\mathrm{T}_{\mathrm{OP}}$ will require: on a $\mathrm{HB}$, warming up to $\mathrm{T}_{0}$ and cooling down to $\mathrm{T}_{\mathrm{OP}}$; on a $\mathrm{CB}$, warming up to above $\mathrm{T}_{\mathrm{M}}$, cooling down to $\mathrm{T}_{0}$, and again warming up to $\mathrm{T}_{\mathrm{OP}}$. If $\mathrm{T}_{\mathrm{OP}}$ is chosen in the middle of a NHB, the last step requires cooling down from $\mathrm{T}_{0}$ to $\mathrm{T}_{0}-\Delta \mathrm{T}_{\mathrm{NHB}} / 2$ on a $\mathrm{HB}$, and warming up from $\mathrm{T}_{0}$ to $\mathrm{T}_{0}+\Delta \mathrm{T}_{\mathrm{NHB}} / 2$ on a $\mathrm{CB}$.

\section{Summary}

We found a regime of operation within a hysteretic phase transition which, within a limited range of a transition-controlling parameter, avoids hysteresis. This may benefit applications utilizing a phase transition but suffering from complications introduced by hysteresis. One such application is IR visualization with resistive microbolometers. We propose to use $\mathrm{VO}_{2}$ as a sensor material, operating it in the non-hysteretic branch (NHB) regime. Partial shorting out of the S-phase by the Mphase lowers sensor resistance; the degree of admixture of M-phase determines the value of this resistance, making it adjustable. At the same time, because of the Sphase percolation, NHB has semiconducting $\mathrm{R}(\mathrm{T})$. The TCR in this case can be considerably higher than in the pure S-phase due to a smooth change of geometry (but not the topology) of S-M areas within an NHB. A similar NHB regime exists in optical reflectivity. Optical reflectivity slopes correlate with TCR-s, revealing the reason for TCR enhancement in some of the NHB-s over the S-phase value. We believe that NHB regime may be implemented in any hysteretic phase transition, removing problems associated with hysteresis and potentially benefiting electronic applications.

\section{Acknowledgements}

We thank Dr. David Westerfeld for developing Lab View-based automated resistivity measurement system and Dr. Arsen Subashiev for useful theoretical discussions. This work was supported by the New York State Foundation for Science, Technology and Innovation (NYSTAR) through the facilities of the Center for Advanced Sensor Technology at Stony Brook. 


\section{References}

1. M. Gurvitch, S. Luryi, A. Polyakov, A. Shabalov, "Non-hysteretic behavior inside the hysteresis loop of $\mathrm{VO}_{2}$ and its possible application in infrared imaging", J. of Appl. Phys. 106(8), (2009).

2. F. J. Morin, Phys. Rev. Letters 3, 34 (1959); N. Mott, "Metal-Insulator Transitions", Taylor \& Francis, London (1997).

3. C. N. Bergland and H. J. Guggenheim, Phys. Rev. 185 (3), pp. 1022-1033 (1969).

4. R. S. Scott and G. E. Frederics, "Model for Infrared Detection by a MetalSemiconductor Phase Transition", Infrared Physics 16, pp. 619-626 (1976).

5. V. Yu. Zerov, Yu. V. Kulikov, V. N. Leonov, V. G. Malyarov, I. A. Khrebtov, and I. I. Shaganov, J. Opt. Technol.66(5), pp. 387-390 (1999).

6. B. E. Cole, R. E. Higashi, and R. A. Wood, Proc. of the IEEE 86(8), p.1679, (1998).

7. T. G. Lanskaya, I. A. Merkulov, and F. A. Chudnovskii, Sov. Phys. Solid State 20(2), 193 (1978) and a number of subsequent references treating hysteresis in $\mathrm{VO}_{2}$.

8. M. Gurvitch, S. Luryi, A. Polyakov, A. Shabalov, M. Dudley, G. Wang, S. Ge, V.Yakovlev, J. Appl. Phys. 102, 033504 (2007). 